\title{
Features of Immunosenescence in Women Newly Diagnosed With Breast Cancer
}

\author{
Lauren Trintinaglia ${ }^{1,2}$, Lucas Poitevin Bandinelli,3, Rodrigo Grassi-Oliveira ${ }^{1,3}$, \\ Laura Esteves Petersen ${ }^{1}$, Marcelo Anzolin', Bruna Luz Correa ${ }^{5}$, \\ Jaqueline Bohrer Schuch ${ }^{1,2}$ and Moisés Evandro Bauer ${ }^{1,2,6 *}$
}

${ }^{1}$ Laboratory of Immunosenescence, School of Sciences, Pontifícia Universidade Católica do Rio Grande do Sul (PUCRS), Porto Alegre, Brazil, ${ }^{2}$ Graduate Program in Biomedical Gerontology, Pontifícia Universidade Católica do Rio Grande do Sul (PUCRS), Porto Alegre, Brazil, ${ }^{3}$ Developmental Cognitive Neuroscience Laboratory (DCNL), School of Health Sciences, Porto Alegre, Brazil, ${ }^{4}$ Centro Universitário Ritter dos Reis, Porto Alegre, Brazil, ${ }^{5}$ Labvitrus Laboratory, Porto Alegre, Brazil, ${ }^{6}$ National Institute of Science and Technology on Neuroimmunomodulation (INCT-NIM), Rio de Janeiro, Brazil

Adults exposed to childhood maltreatment have increased stress reactivity. This profile is associated with dysregulation of the immune system, including enhanced inflammatory reactions and accelerated senescence. Subjects exposed to ear stress have increased risk for several age-related diseases, including cardiovascular disease, type II diabetes, and cancer. Although previous studies have reported immune changes in advanced cancer, very little information is available regarding early stage breast cancer. Here, 29 patients with breast cancer were recruited: 15 with history of childhood maltreatment $(\mathrm{CM}+)$ and 14 without history (CM-). Twenty-seven healthy women without $\mathrm{CM}$ were selected as the control group. Peripheral blood was collected and lymphocyte subsets phenotyped by multi-color flow cytometry (B cells, CD4+ T, CD8+ T, natural killer cells, activated T cells, regulatory T cells, and senescence-associated T cells). Because human cytomegalovirus (CMV) was associated with signatures of early senescence, the CMV serology was determined by ELISA. None of the subjects had lgM reactivity to CMV, excluding acute viral infection. There was a higher proportion of patients with increased CMV IgG levels in the CM+ group as compared to $\mathrm{CM}$ - or controls. Different stages of T-cell differentiation can be determined based on the cell-surface expression of the costimulatory molecules CD27 and CD28: ear (CD27+CD28+), intermediate-differentiated (CD27-CD28+), and late-differentiated or senescent $T$ cells (CD27-CD28-). After adjusting for age and education, ear T cells (CD27+CD28+) were found reduced in $\mathrm{CM}+$ and $\mathrm{CM}$ - patients $(p<0.0001)$. In contrast, intermediate-differentiated T cells (CD27-CD28+; $p<0.0001)$, senescent T cells (CD27-CD28-; $p<0.0001)$, and exhausted T cells (CD8+CD27-CD28-PD1+; $p<0.0001)$ were found expanded in both $\mathrm{CM}+$ and $\mathrm{CM}-$ groups. Our data suggest that features of immunosenescence are associated with newly diagnosed breast cancer, regardless of the CM history.

\section{Keywords: childhood maltreatment, breast cancer, immunosenescence, cytomegalovirus, T lymphocytes}

\section{INTRODUCTION}

Childhood maltreatment, such as abuse and neglect, increases the vulnerability for the development of psychiatric disorders and cancer in adult life $(1,2)$. A large meta-analysis revealed that having multiple adverse childhood experiences was associated with increased risk $(\mathrm{OR}=2.3)$ for development of cancer (3). Adults with history of early life stress have increased reactivity of the stress system, with altered cortisol responses to psychosocial stressors $(4,5)$. A new cancer diagnosis is associated 
with important psychological burden and can be understood as a "second allostatic hit" (6), further activating the stress system (7). This profile is also associated with dysregulation of the immune system, mainly characterized by increased levels of proinflammatory cytokines and early senescence (8-11). Therefore, the understanding of biological changes associated with early life stress at the cancer onset is of paramount importance. The monitoring of these biomarkers would be beneficial for planning therapies and optimizing the timing of treatment.

Recent evidence indicated that chronic stress in adults as well as childhood maltreatment may lead to accelerated aging of the immune system (immunosenescence) (12). This was demonstrated by shortened telomeres, and changes in specific lymphocyte populations, including the expansion of $\mathrm{T}$ cells with late differentiated profile, and increased senescence and exhaustion cellular markers (e.g., PD1 and KLRG1, respectively) $(13,14)$. Of note, different stages of T-cell differentiation can be determined based on the cell-surface expression of the costimulatory molecules CD27 and CD28 $(15,16)$. Previous studies have defined naïve $\mathrm{T}$ cells or early differentiated (CD27+CD28+), intermediatedifferentiated (CD27-CD28+), and late-differentiated or senescent cells (CD27-CD28-). Furthermore, previous studies suggested that childhood maltreatment was associated with increased serology to cytomegalovirus (CMV) (17), as similarly reported in aging studies $(12,18)$. The immunosenescence profile is largely unknown in patients with breast cancer, and it is speculated these changes may interfere in the prognosis and treatment response.

In this study, we investigated the presence of immunosenescence markers (lymphocyte subtypes and CMV serology) in women newly diagnosed with breast cancer with and without history of childhood maltreatment.

\section{MATERIALS AND METHODS}

\section{Subjects}

Twenty-nine women with early breast cancer stage (stages $0-3 \mathrm{~A}$ ) were recruited before starting treatment (chemotherapy, radiotherapy, or surgery) from the Mastology Unit at São Lucas Hospital, PUCRS (Porto Alegre, Brazil). Patients with history of major depression, inflammatory or immune-based diseases, as well as a prior history of breast cancer or presence of others cancers were excluded. Clinical characteristics were assessed through hospital medical records and/or questionnaires. In addition, 36 women without breast cancer were selected as the control group - nine of them were excluded due to history of childhood abuse or neglect.

Childhood maltreatment was investigated by the Childhood Trauma Questionnaire-Portuguese version (19). CTQ is a retrospective 28-item self-report instrument that assesses exposure to sexual, physical and emotional abuse, and physical and emotional neglect (20). In this study, the group with childhood maltreatment $(\mathrm{CM}+)$ consisted of participants who reported at least one moderate or severe type of childhood abuse or neglect. The group without childhood maltreatment $(\mathrm{CM}-)$ consisted of participants who reported none or low scores of CM. Among the patients, 15 were selected as $\mathrm{CM}+$ and 14 were included in the CM- group. The healthy controls had no history of abuse or neglect. Cases and controls were evaluated for depressive symptoms using the Beck Depression Inventory-Portuguese version (BDI-II) (21). The study protocol was approved by both scientific and ethics committees of PUCRS (Porto Alegre, Brazil) and written informed consent was obtained from all participants.

\section{Isolation of Peripheral Blood Mononuclear Cells (PBMCs)}

Peripheral blood $(10 \mathrm{~mL})$ was collected between 10 and $12 \mathrm{~h}$ from each participant by venipuncture in EDTA tubes. Plasma was isolated and stored at $-80^{\circ} \mathrm{C}$. PBMCs were isolate by Ficoll density gradient centrifugation (Ge Healthcare Life SciencesMarlborough, MA, USA), $30 \mathrm{~min}$ at $900 \mathrm{~g}$. Cells were counted using a microscope $(100 \times)$ and viability always exceeded $95 \%$, as judged by Trypan Blue exclusion (Sigma-Aldrich-St. Louis, MO, USA).

\section{Immunophenotyping}

A comprehensive panel of lymphocyte subsets was identified by multicolor flow cytometry. Briefly, PBMCs were washed in flow cytometry buffer (PBS containing 1\% FCS and 0.01\% sodium azide) and treated with Fc Block solution for $20 \mathrm{~min}$. Cells were stained for $30 \mathrm{~min}$ at $4^{\circ} \mathrm{C}$ with combinations of monoclonal antibodies: anti-CD3 FITC (T cells), anti-CD4 PECy5, FITC and APC (Th cells), anti-CD8 PECy5 (Tc cells), anti-CD19 APC (B cells), anti-CD56 APC [natural killer (NK) cells], anti-CD57 FITC (NK), anti-CD28 APC, anti-CD27 PE, anti-CD69 FITC (early activated cells), anti-CD25 FITC (early activated cells), anti-CD103 FITC (regulatory T cell marker), anti-NKG2 (senescent marker), antiKLRG1 (senescent marker), and anti-PD1 (exhaustion marker). The differentiation stages of $\mathrm{T}$ cells were studied as the following criteria: CD27+CD28+ (early differentiated), CD27-CD28+ (intermediate-differentiated), and CD27-CD28- (senescent cells).

All antibodies were purchased from BD Biosciences (San Jose, CA, USA), except anti-NKG2 (Bio-Techne, Minneapolis, MN, USA), anti-KLRG1 (Biolegend, San Diego, CA, USA), and antiPD1 (Biolegend, San Diego, CA, USA). After staining, cells were washed, resuspended, and analyzed by flow cytometry. At least 20,000 lymphocytes were identified by size (FSC) and granularity (SSC) and acquired using a FACS Canto II flow cytometer (BD Biosciences). The instrument was checked for sensitivity and overall acquisition. Data were analyzed using Flowjo 7.2.5 software (Tree Star Inc., Ashland, OR, USA).

\section{Serology}

Plasma samples were analyzed for both IgM and IgG antibodies anti-CMV using enzyme-linked immunosorbent assays (ELISAs) (IBL International, Hamburg, Germany). Sensitivity and specificity were estimated to be more than $95 \%$. The optical densities $(570 / 620 \mathrm{~nm})$ were estimated in an ELISA plate reader. Samples were considered positive (reactive) for antibodies anti-CMV when values were above the cut-off of $22 \mathrm{IU} / \mathrm{mL}$ for IgG. The detection limit is $0.4 \mathrm{IU} / \mathrm{mL}$ for both IgM and IgG anti-CMV. The results are expressed in International units per milliliter. 


\section{Statistical Analyses}

All variables were tested for normality of distribution by ShapiroWilk tests. For continuous variables, differences between groups (CM+, $\mathrm{CM}-$, and controls) were evaluated by Analysis of Variance (ANOVA) or Kruskal-Wallis (K-W). For categorical variables, differences between groups were compared using chi-square $\left(X^{2}\right)$ test. Generalized Linear Modeling (GzLM) was also used to compare differences between groups adjusting for potential confounders (age and education). Linear or gamma distribution was selected based on the distribution outcome and robust estimation with unstructured working correlation matrix was set. Bonferroni post hoc test was used to compare means between groups and adjust the observed significance level considering multiple contrast being tested. Relationship between continuous variables were analyzed by Pearson or Spearman's correlation tests. Statistical analyses were performed using the Statistical Package for Social Sciences, SPSS Statistics V.20 software (SPSS Inc., Chicago, IL, USA). The significance level was set at $\alpha=0.05$ (two tailed).

\section{RESULTS}

\section{Sociodemographic and Clinical Characteristics}

Demographic and clinical characteristics of the samples are summarized in Table 1. All groups were similar regarding BDI scores. Individuals of $\mathrm{CM}+$ and $\mathrm{CM}-$ groups differed from control group by age, years of education, and income (all $p<0.05$ ). $\mathrm{CM}+$ and $\mathrm{CM}-$ groups present similar cancer stage and family history of breast cancer. As expected, higher CTQ scores were observed in the $\mathrm{CM}+$ group compared to $\mathrm{CM}-$ and control groups $(\mathrm{K}-\mathrm{W}=28.4, p<0.001)$.

\section{Major Lymphocyte Subsets}

We investigated different peripheral lymphocyte subpopulations associated with activation and regulatory profiles (Table 2). Activated $\mathrm{T}$ cells $(\mathrm{CD} 3+\mathrm{CD} 69+)$ and regulatory $\mathrm{T}$ cells $(\mathrm{CD} 4+\mathrm{CD} 103+)$ were found significantly increased in $\mathrm{CM}+$ and $\mathrm{CM}-$ patients (all $p<0.0001$ ) compared to controls. Figure 1

TABLE 1 | Demographic and clinical data of studied groups.

\begin{tabular}{|c|c|c|c|c|c|}
\hline & Controls $(n=27)$ & $\mathrm{CM}+(n=15)$ & $C M-(n=14)$ & Statistics & Pairwise comparison \\
\hline Age (years) & $40.3 \pm 10.3^{a}$ & $50.4 \pm 9.9^{b}$ & $49.4 \pm 11.8^{c}$ & $F=5.9, p=0.005$ & $b$ and $c>a$ \\
\hline Education (years) & $18.0 \pm 4.0^{\mathrm{a}}$ & $9.9 \pm 4.31^{\mathrm{b}}$ & $13.7 \pm 5.13^{c}$ & $F=15.2, p<0.001$ & $\mathrm{~b}$ and $\mathrm{c}<\mathrm{a}$ \\
\hline Income, monthly (US\$) & $2,364.6 \pm 357.7^{a}$ & $686.4 \pm 100.1^{b}$ & $502.9 \pm 95.7^{c}$ & $\mathrm{~K}-\mathrm{W}=28.4, p<0.001$ & $\mathrm{~b}$ and $\mathrm{c}<\mathrm{a}$ \\
\hline Ethnicity (\% Caucasian) & $26(96.3)$ & $10(66.7)$ & $10(71.4)$ & $X^{2}=6.2, p=0.04$ & \\
\hline Family history (yes) & - & $5(35.7)$ & $8(61.5)$ & $X^{2}=1.8, p=0.40$ & \\
\hline \multicolumn{6}{|l|}{ Cancer stage } \\
\hline Stage I & - & 7 & 5 & & \\
\hline Stage ॥ & - & 2 & 5 & $X^{2}=1.8, p=0.40$ & \\
\hline Stage III & - & 6 & 4 & & \\
\hline BDI-II & $6.2 \pm 4.0$ & $9.1 \pm 5.5$ & $8.3 \pm 6.3$ & $F=1.7, p=0.19$ & \\
\hline CTQ & $29.1 \pm 0.8^{a}$ & $53.5 \pm 3.4^{b}$ & $29.9 \pm 1.1^{\circ}$ & $\mathrm{K}-\mathrm{W}=28.4, p<0.001$ & $a$ and $c<b$ \\
\hline
\end{tabular}

Data shown as mean $\pm S D$.

$\mathrm{CM}+$, breast cancer women with history of childhood Maltreatment; CM-, breast cancer women without history of childhood maltreatment; BDI, Beck Depression Inventory; CTQ, Childhood Trauma Questionnaire.

F, Analysis of Variance; $X^{2}$, chi-squared; K-W, Kruskal-Wallis.

TABLE 2 | Immunophenotyping of major lymphocyte subsets.

\begin{tabular}{|c|c|c|c|c|c|c|}
\hline Markers (\%) & Cell type & Controls & $\mathrm{CM}+$ & CM- & Statistics (Wald) & $p$-Value \\
\hline CD3+CD4+ & Th & $45.4 \pm 2.5^{\mathrm{a}}$ & $41.1 \pm 3.7$ & $33.0 \pm 2.3^{b}$ & 14.2 & $<0.001$ \\
\hline CD4/CD8 & Ratio & $2.2 \pm 0.2$ & $2.3 \pm 0.4$ & $1.6 \pm 0.1$ & 4.5 & 0.106 \\
\hline $\mathrm{CD} 3+\mathrm{CD} 8+$ & Tc & $23.7 \pm 1.6$ & $21.3 \pm 3.2$ & $24.1 \pm 2.7$ & 0.4 & 0.798 \\
\hline CD3-CD19+ & $\mathrm{B}$ & $13.8 \pm 0.9$ & $10.0 \pm 1.2^{\mathrm{a}}$ & $15.4 \pm 1.8^{b}$ & 7.7 & 0.021 \\
\hline CD3-CD56+ & NK & $8.9 \pm 0.8$ & $8.3 \pm 1.0$ & $7.1 \pm 0.8$ & 2.2 & 0.327 \\
\hline CD3+CD56+ & NK T & $6.6 \pm 0.7$ & $4.6 \pm 0.7$ & $6.2 \pm 1.1$ & 2.6 & 0.271 \\
\hline $\mathrm{CD} 3+\mathrm{CD} 57+$ & NK & $11.8 \pm 1.3$ & $7.2 \pm 1.6$ & $10.6 \pm 1.6$ & 3.8 & 0.145 \\
\hline CD3+CD4+CD25+ & Activated T cell & $2.1 \pm 0.1$ & $2.6 \pm 0.1$ & $2.4 \pm 0.1$ & 2.7 & 0.251 \\
\hline CD3+CD8+CD25+ & Activated T cell & $0.6 \pm 0.1$ & $0.8 \pm 0.2$ & $0.7 \pm 0.1$ & 2.1 & 0.346 \\
\hline CD3+CD69+ & Activated T cell & $1.3 \pm 0.1^{\mathrm{a}}$ & $1.9 \pm 0.1^{b}$ & $1.8 \pm 0.1^{b}$ & 23.3 & $<0.0001$ \\
\hline CD4+CD103+ & Regulatory $T$ cell & $0.4 \pm 0.2^{\mathrm{a}}$ & $1.3 \pm 0.1^{b}$ & $1.4 \pm 0.1^{b}$ & 194.8 & $<0.0001$ \\
\hline CD8+CD103+ & Regulatory $T$ cell & $0.7 \pm 0.1$ & $0.7 \pm 0.2$ & $0.6 \pm 0.2$ & 0.8 & 0.663 \\
\hline
\end{tabular}

Data shown as mean percentage \pm SE. Data were analyzed by Generalized Linear Modeling test (gamma or linear distribution) adjusted for age and years of education. Statistical significant differences are highlighted in bold font.

a,b Differences between groups (Bonferroni post hoc test).

$\mathrm{CM}+$, breast cancer women with history of childhood maltreatment; CM-, breast cancer women without history of childhood maltreatment; Th, helper T cell; Tc, cytotoxic T cell; NK, natural killer cell. 
shows the mean differences of activation/regulatory markers between patients and controls. Furthermore, the $\mathrm{CM}+$ group had reduced frequencies of CD3-CD19+ B cells $(p=0.021)$ compared to CM- group. Decreased proportions of helper T cells $(\mathrm{CD} 3+\mathrm{CD} 4+)$ were observed in the CM- group $(p<0.001)$, but not in the $\mathrm{CM}+$ group or controls. The CD4/CD8 ratio did not differ between groups $(p=0.10)$. No significant differences were found between groups for the remaining subpopulations.

\section{Lymphocyte Subsets With Senescence \\ Profile}

Different stages of T-cell differentiation can be described based on the expression of cell-surface co-stimulatory molecules CD27 and CD28 $(15,16)$ (Table 3$)$. The early differentiated T cells $(\mathrm{CD} 4+\mathrm{CD} 27+\mathrm{CD} 28+$ and $\mathrm{CD} 8+\mathrm{CD} 27+\mathrm{CD} 28+)$ were found significantly reduced in $\mathrm{CM}+$ and $\mathrm{CM}-$ patients (all $p<0.0001)$ compared to controls. In contrast, the intermediate-differentiated T cells (CD4+CD27-CD28+), and late-differentiated (senescent) T cells (CD4+CD27-CD28-) were found expanded in patients (all $p<0.0001$ ). The Figure 2 shows the shrinkage of the early differentiated T-cell pool in contrast to the expansion of the pool of late-differentiated $\mathrm{T}$ cells in patients.

Similarly, the senescent T cell CD8+KLRG1+NKG2+ was found expanded in both $\mathrm{CM}+$ and $\mathrm{CM}-$ patients $(p<0.0001)$. Also, the early senescent T cell CD3+CD8+KLRG1+ was found significantly increased in the $\mathrm{CM}-$ but not in $\mathrm{CM}+$ group or controls $(p=0.002)$. The CD8+CD27-CD28-PD1+ T cells

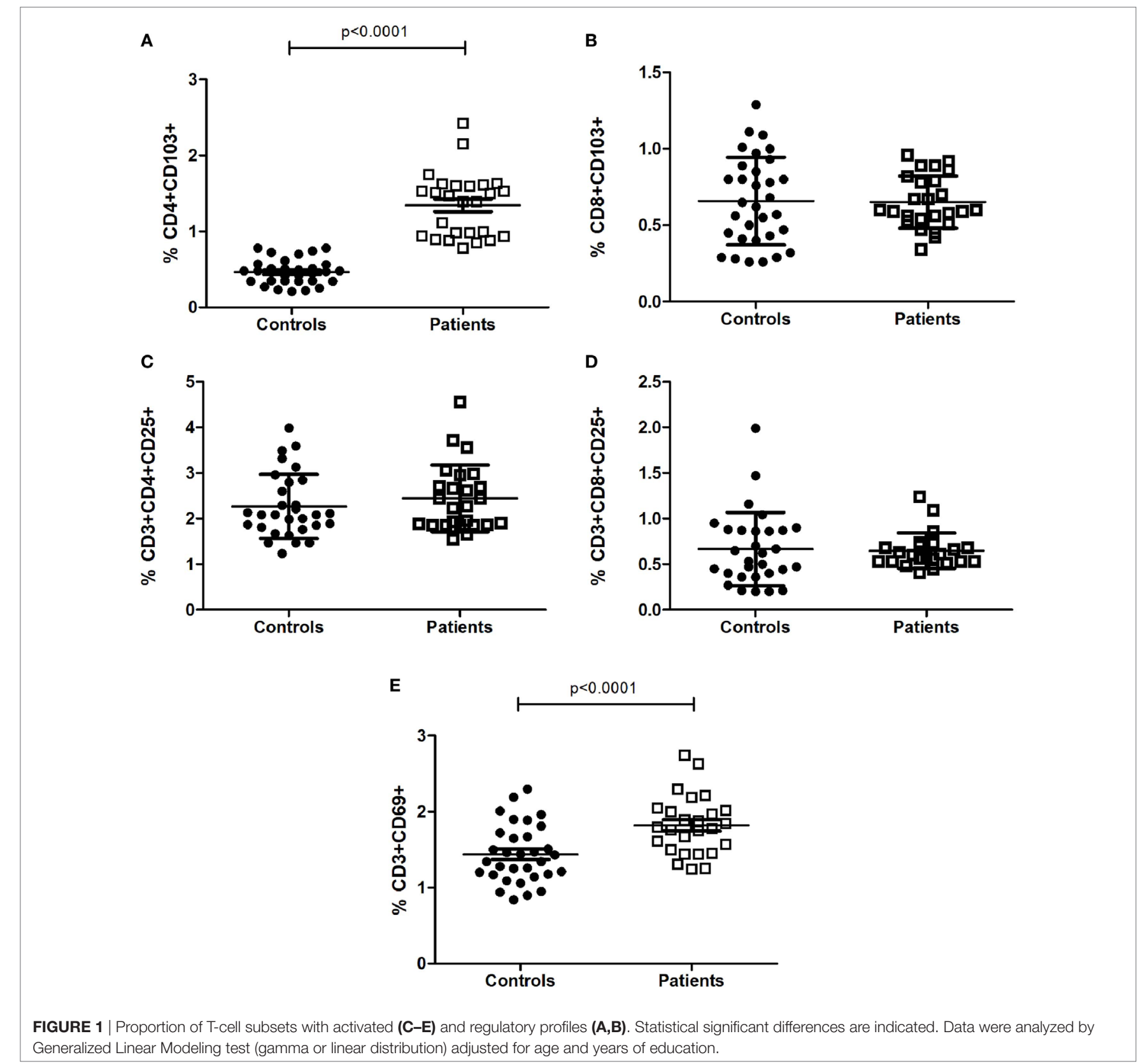


TABLE 3 | Different stages of T-cell differentiation and senescence-related markers.

\begin{tabular}{|c|c|c|c|c|c|c|}
\hline Markers (\%) & Cell type & Controls & $\mathrm{CM}+$ & CM- & Statistics (Wald) & $p$-Value \\
\hline $\mathrm{CD} 4+\mathrm{CD} 27+\mathrm{CD} 28+$ & Early differentiated T cell & $42.0 \pm 4.6^{a}$ & $17.5 \pm 3.9^{b}$ & $19.2 \pm 3.9^{b}$ & 17.6 & $<0.0001$ \\
\hline CD8+CD27+CD28+ & Early differentiated $\mathrm{T}$ cell & $27.9 \pm 3.3^{a}$ & $8.7 \pm 1.2^{\mathrm{b}}$ & $12.0 \pm 2.9^{b}$ & 38.1 & $<0.0001$ \\
\hline CD4+CD27-CD28+ & Intermediate-differentiated T cell & $8.74 \pm 1.1^{\mathrm{a}}$ & $25.0 \pm 8.8^{b}$ & $25.7 \pm 8.2^{b}$ & 12.9 & 0.002 \\
\hline CD8+CD27-CD28+ & Intermediate-differentiated T cell & $7.1 \pm 0.9$ & $6.9 \pm 2.1$ & $8.0 \pm 1.8$ & 0.2 & 0.880 \\
\hline CD4+CD27-CD28- & Late-differentiated T cell & $13.9 \pm 1.2^{\mathrm{a}}$ & $21.8 \pm 2.1^{\mathrm{b}}$ & $22.9 \pm 1.5^{b}$ & 19.8 & $<0.0001$ \\
\hline CD8+CD27-CD28- & Late-differentiated T cell & $39.4 \pm 3.4$ & $34.4 \pm 4.6$ & $42.8 \pm 5.5$ & 1.6 & 0.431 \\
\hline CD3+CD56+NKG2+ & Senescent NK T cell & $0.5 \pm 0.1$ & $0.6 \pm 0.1$ & $0.7 \pm 0.3$ & 3.4 & 0.179 \\
\hline $\mathrm{CD} 3+\mathrm{CD} 4+\mathrm{KLRG} 1+$ & Senescent T cell & $5.1 \pm 0.8$ & $10.7 \pm 3.0$ & $11.5 \pm 4.0$ & 3.4 & 0.183 \\
\hline CD3+CD8+KLRG1+ & Senescent $\mathrm{T}$ cell & $8.7 \pm 0.9$ & $6.1 \pm 0.9^{a}$ & $9.3 \pm 1.5^{b}$ & 8.7 & 0.013 \\
\hline CD4+KLRG1+NKG2+ & Senescent $\mathrm{T}$ cell & $5.96 \pm 2.8$ & $19.0 \pm 4.0$ & $21.0 \pm 4.5$ & 5.4 & 0.065 \\
\hline CD8+KLRG1+NKG2+ & Senescent $\mathrm{T}$ cell & $6.0 \pm 0.8^{a}$ & $20.0 \pm 3.9^{b}$ & $24.9 \pm 7.7^{b}$ & 17.1 & $<0.0001$ \\
\hline CD8+CD27-CD28-PD1+ & Exhausted T cell & $7.6 \pm 2.5^{a}$ & $78.3 \pm 0.10 .0^{b}$ & $97.8 \pm 9.7^{\mathrm{b}}$ & 55.5 & $<0.0001$ \\
\hline
\end{tabular}

Data shown as mean percentage \pm SE. Data were analyzed by Generalized Linear Modeling test (gamma or linear distribution) adjusted for age and years of education. Statistical significant differences are highlighted in bold font.

a,b Differences between groups (Bonferroni post hoc test).

$\mathrm{CM}+$, breast cancer women with history of childhood maltreatment; CM-, breast cancer women without history of childhood maltreatment; NK, natural killer cell.

\section{CD4+ $\mathrm{T}$ cell differentiation (Controls)}

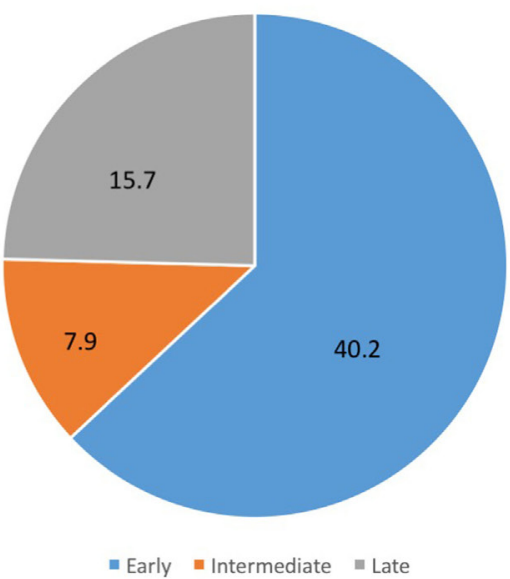

CD8+ $\mathrm{T}$ cell differentiation (Controls)

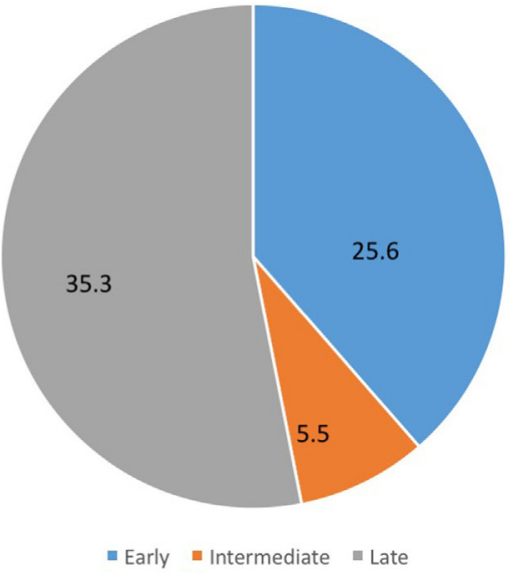

CD4+ T cell differentiation (Patients)

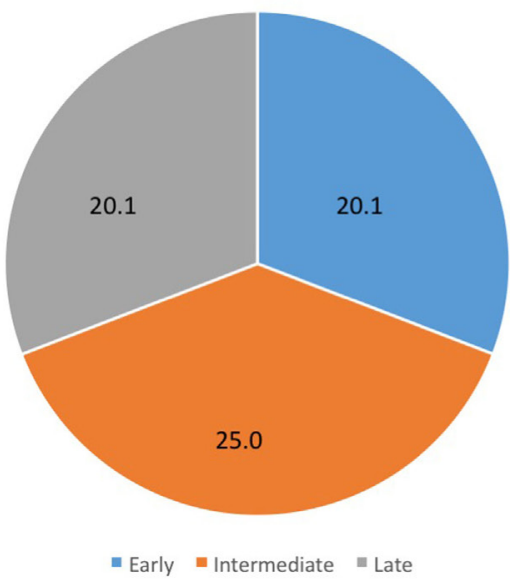

CD8+ T cell differentiation (Patients)

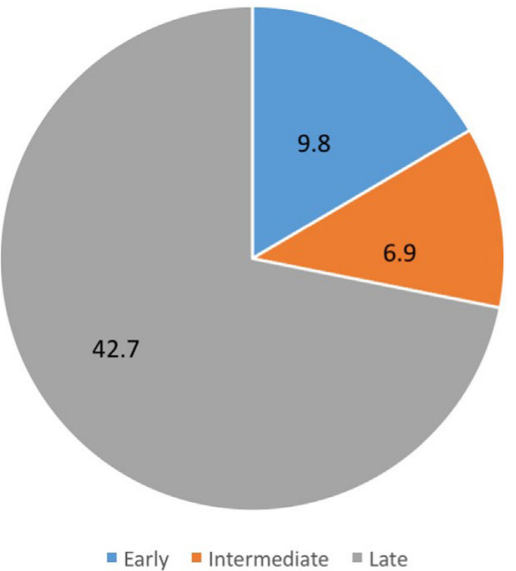

FIGURE 2 | Different stages of T-cell differentiation between patients and controls. The following stages of differentiation can be described based on the expression of cell-surface co-stimulatory molecules CD27 and CD28 (15, 16): early differentiated (CD27+CD28+), intermediate-differentiated (CD27-CD28+) and latedifferentiated (CD27-CD28-) T cells. The figure shows the shrinkage of the early differentiated T-cell pool in contrast to the expansion of the pool of latedifferentiated $\mathrm{T}$ cells in patients. 
(exhausted T cells) were found expanded in both $\mathrm{CM}+$ and $\mathrm{CM}-$ groups when compared to controls $(p<0.0001)$.

\section{Expression Levels of Cell-Surface Markers}

Next, we investigated the expression levels of the studied cell-surface markers by the analysis of the mean fluorescence intensity (MFI), an estimation of the receptor density (Table 4). Of note, we assessed the following biomarkers: CD3, CD19, CD69, CD103, CD27, CD28, KLRG1, NKG2, and PD-1. The $\mathrm{CM}+$ group had higher expression of CD103 in CD4 T cells $(p<0.0001)$ and of NKG2 in CD8 T cells $(p<0.0001)$ as compared to $\mathrm{CM}-$ or control groups. Furthermore, the CD27 expression in CD4 T cells was found higher in controls compared to $\mathrm{CM}+$ and $\mathrm{CM}-$ groups $(p=0.002)$. In CD8 T cells, higher CD27 and CD28 expression was observed in controls compared to women with breast cancer $(p=0.001$ and $p<0.0001$, respectively).

\section{Influence of CMV on Antibody Titers}

The CMV serology was investigated here as another index of accelerated immunosenescence. None of the subjects had $\operatorname{IgM}$ reactivity to $\mathrm{CMV}$, excluding acute viral infection. The median IgG anti-CMV titers was 114.40, and there was a higher proportion of subjects with increased serology ( $>$ median) in the CM+ group $(n=12)$ as compared to CM- $(n=7)$ and controls $(n=4), \chi^{2}=14.67, p<0.001$ (Figure 3A). The CM+ patients had increased CMV IgG levels [median $=141.0$ (interquartile range; IQR) (124.7-276.0)] as compared to controls $[$ median $=94.1(85.7-111.2)], p<0.05$ (Figure 3B). However, the CM- group had similar CMV IgG levels [median $=126.9$ (109.0-137.1)] as compared to $\mathrm{CM}+(p=0.22)$ or control groups $(p=1.0)$. As expected, age $\left(r_{\mathrm{s}}=0.52, p<0.0001\right)$ and education $\left(r_{s}=-0.58, p<0.0001\right)$ were correlated to CMV IgG levels (Figures 3C,D). Therefore, when adjusted for these confounders, the CMV IgG levels did not differ between groups (Wald $=0.27, p=0.87$ ).

As there were no differences in CMV IgG levels between CM+ and $\mathrm{CM}-$ groups, the following analyses were thus performed without considering childhood maltreatment. The relationships between CMV IgG levels and lymphocyte subsets were analyzed separately in controls and breast cancer patients. In women with breast cancer, positive correlations were found between anti CMV IgG levels and percentage of CD4+CD27-CD28$\left(r_{\mathrm{s}}=0.71, p<0.0001\right.$, Figure 4A), CD8+CD27-CD28$\left(r_{\mathrm{s}}=0.44, p=0.04\right.$, Figure 4B), NK-T cells $\left(r_{\mathrm{s}}=0.51, p=0.02\right.$, Figure 4C), and NK cells ( $r_{\mathrm{s}}=0.56, p=0.007$, Figure 4D). In the control group, positive correlations were detected between anti CMV IgG levels and the following cell subtypes: CD3+CD56+ $\left(r_{\mathrm{s}}=0.57, p<0.001\right), \mathrm{CD} 4+\mathrm{CD} 27+\mathrm{CD} 28+\left(r_{\mathrm{s}}=0.43, p=0.01\right)$, $\mathrm{CD} 8+\mathrm{CD} 27+\mathrm{CD} 28+\left(r_{\mathrm{s}}=0.49, p=0.06\right), \mathrm{CD} 4+\mathrm{CD} 27-\mathrm{CD} 28+$ $\left(r_{\mathrm{s}}=0.42, p=0.01\right)$, and CD8+CD27-CD28+ $\left(r_{\mathrm{s}}=0.39\right.$, $p=0.03$ ) (Figure 5). In addition, negative correlations between $\mathrm{CD} 3+\mathrm{CD} 103+\left(r_{\mathrm{s}}=-0.47, p=0.09\right)$ and CD4+CD27-CD28$\left(r_{\mathrm{s}}=-0.44, p=0.02\right)$ with anti-CMV IgG levels were also observed.

\section{DISCUSSION}

In this study, we observed the presence of a cellular senescence profile in women with breast cancer, regardless of the history of childhood maltreatment. Very little is known about the effects of early life stress on subjects diagnosed with cancer. A prospective study with women with breast cancer explored the effects of childhood adversity on depressive symptoms and immune functions over 37 weeks following breast surgery (22). At initial assessment, women exposed to $\mathrm{CM}$ had greater perceived stress, depressive symptoms, as well as lower NK cell activity (NKCA). In that study, clinical features concerning treatment variables (radiation therapy, surgery, time since surgery, use of hormonal treatment), disease severity (ongoing cancer), and CTQ scoring may explain the reported associations between CM and low NKCA. In our study, newly diagnosed patients were recruited (before any treatment) and no differentiation in CTQ scoring could be made because of limited sampling in subscales. The presence of CM, of note, the sexual abuse, has been associated with

TABLE 4 | Expression of activated and regulatory markers as determined by the mean fluorescence intensity (MFI).

\begin{tabular}{|c|c|c|c|c|c|}
\hline Markers & Controls & $\mathrm{CM}+$ & CM- & Statistics (Wald) & $p$-Value \\
\hline \multicolumn{6}{|l|}{ CD3+ } \\
\hline CD19 & $4,894 \pm 645$ & $4,439 \pm 871$ & $4,128 \pm 948$ & 0.47 & 0.790 \\
\hline CD69 & $358 \pm 33$ & $487 \pm 78$ & $423 \pm 65$ & 3.01 & 0.222 \\
\hline \multicolumn{6}{|l|}{ CD4+ } \\
\hline CD103 & $384 \pm 33^{a}$ & $854 \pm 139^{a, b}$ & $568 \pm 95^{b}$ & 19.92 & $<0.0001$ \\
\hline CD27 & $4,024 \pm 244^{a, b}$ & $3,088 \pm 200^{a}$ & $2,869 \pm 286^{b}$ & 12.84 & 0.002 \\
\hline CD28 & $3,109 \pm 316$ & $2,638 \pm 351$ & $2,171 \pm 281$ & 4.78 & 0.091 \\
\hline \multicolumn{6}{|l|}{ CD8+ } \\
\hline KLRG1 & $2,638 \pm 237$ & $2,964 \pm 383$ & $2,897 \pm 95$ & 0.95 & 0.954 \\
\hline NKG2 & $1,746 \pm 334^{a}$ & $3,893 \pm 344^{a, b}$ & $1,603 \pm 377^{b}$ & 26.9 & $<0.0001$ \\
\hline PD-1 & $2,667 \pm 123$ & $2,876 \pm 154$ & $2,956 \pm 165$ & 1.67 & 0.756 \\
\hline CD27 & $3,965 \pm 223^{a, b}$ & $2,978 \pm 265^{a}$ & $2,804 \pm 290^{b}$ & 13.6 & 0.001 \\
\hline CD28 & $2,543 \pm 215^{a, b}$ & $1,217 \pm 118^{a}$ & $1,489 \pm 177^{b}$ & 25.3 & $<0.0001$ \\
\hline
\end{tabular}

Data shown as mean MFI \pm SE. Data were analyzed by Generalized Linear Modeling (linear or gamma distribution) adjusted for age and education years.

Statistical significant differences are highlighted in bold font.

a,b Differences between groups (Bonferroni post hoc test). 

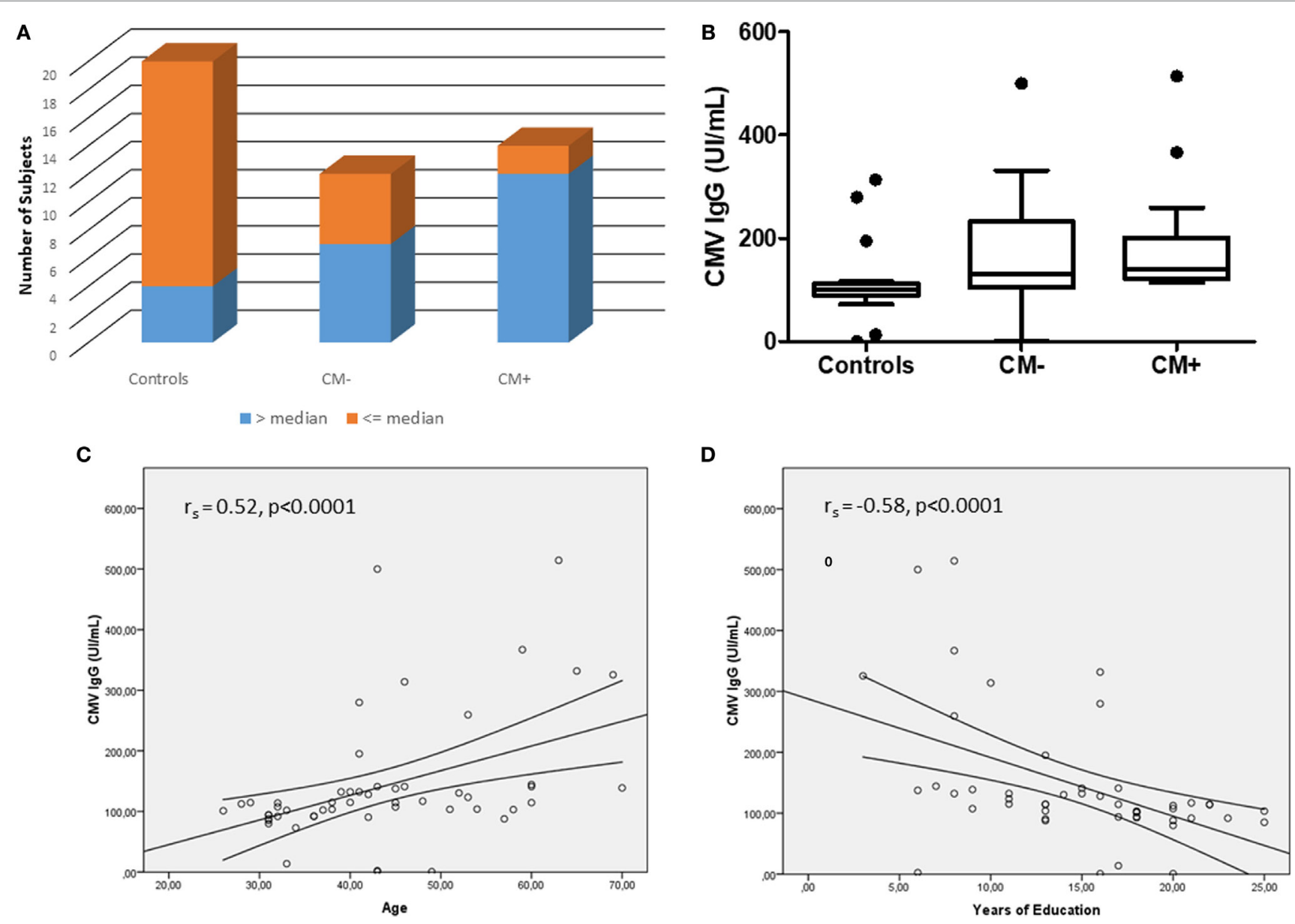

FIGURE 3 | The cytomegalovirus (CMV) serology between studied groups. (A) Shows the number of subjects with lgG anti-CMV levels > or $\leq \mathrm{median}$ (114.40). (B) Shows the CMV IgG levels between groups. After adjusting for age and years of education, no differences were observed between groups (Wald = 0.27 , $p=0.87)$. (C,D) Show the correlations between CMV IgG serology and age and education, respectively.

increased incidence of adulthood cancer (23). Therefore, we speculate that early life stress and disease progression (e.g., treatment) may participate in the "second hit model" (6) to promote synergistically allostatic changes, including those involving the immune system.

Previous studies in the field have described immune changes in breast cancer, usually involving immunotherapeutic aspects and response to treatment. The frequency of activated T-cells is modulated by the presence of tumors, as demonstrated in a study comparing women with breast cancer and healthy controls $(22,24)$. As observed in our study, there was an increase in the proportion of activated T cells (CD69+) in breast cancer. B cells also seem to be expanded in women with breast cancer (25). We observed a similar increase in the proportion of B cells in the group of women with breast cancer who were not exposed to childhood abuse when compared to healthy controls.

In our study, we observed distinct changes involving aging lymphocytes. Of note, there was an expansion of CD27-CD28- T cells (late-differentiated or senescent T cells) in contrast to reduced proportion of CD28+CD27+ T cells (early differentiated). The presence of the T-cell senescent profile is corroborated by the loss of the CD27 costimulatory molecule, as shown here by reduced CD27 expression, as estimated by the MFI. Similarly, an increase in the proportion of CD8+CD28- T cells was observed in women with breast cancer during chemotherapy (26), and in lung cancer (27). Previous studies have also reported that the increase of this lymphocyte type in cancer patients may be associated with the advanced staging and treatment inefficacy, since some immunotherapeutic interventions require the presence of this costimulatory molecule to be effective. In addition, the expansion of this population of senescent cells can lead to an inefficient antitumor response, due to the decrease of naïve and effector T-cells $(26,28,29)$. However, in our study, the staging of the disease was similar between the cases, and it was not possible to evaluate the potential involvement of the lymphocyte subtypes with the clinical outcome.

In their terminal differentiation (i.e., senescence), T-cells also express regulatory markers such as KLRG1 and PD-1 (13). Exposure to chronic stress in childhood can affect the 

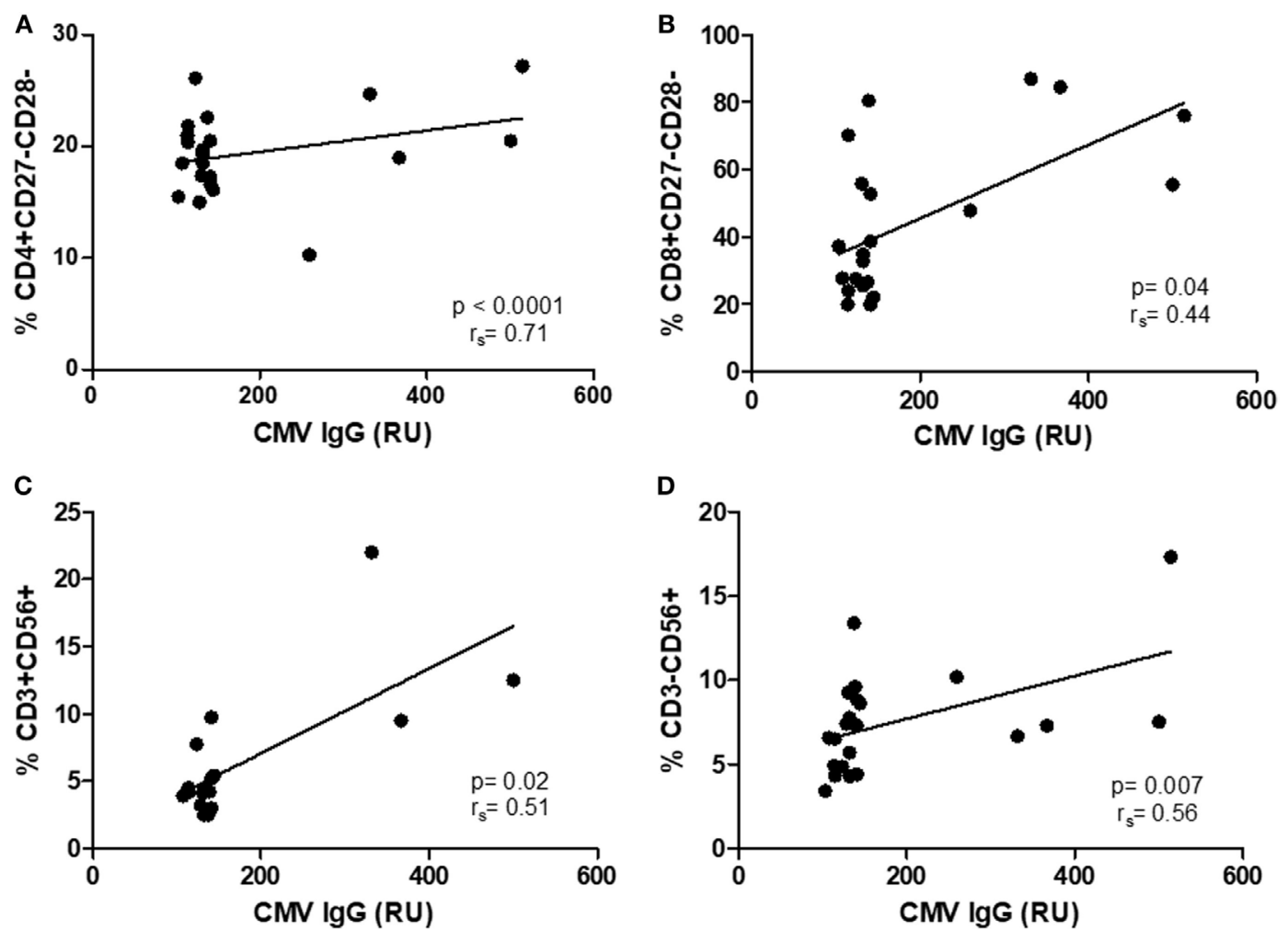

FIGURE 4 | Correlation between IgG anti-cytomegalovirus (CMV) titers and immunosenescence markers in women with breast cancer. (A-D) Show the correlations between senescent CD4 and CD8 T cell $\left(r_{\mathrm{s}}=0.71, p<0.0001 ; r_{\mathrm{s}}=0.44, p=0.04\right)$ and natural killer and NKT $\left(r_{\mathrm{s}}=0.51, p=<0.02 ; r_{\mathrm{s}}=56, p=0.007\right)$ and anti-CMV titers.

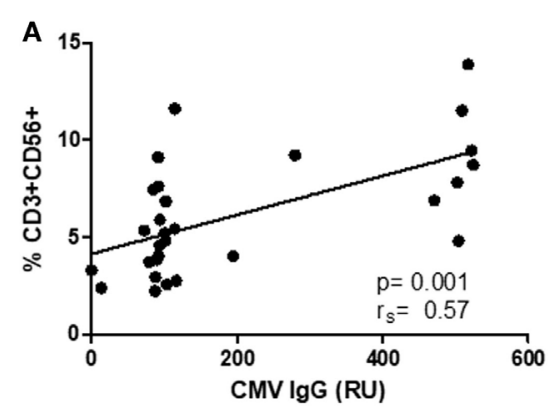

D

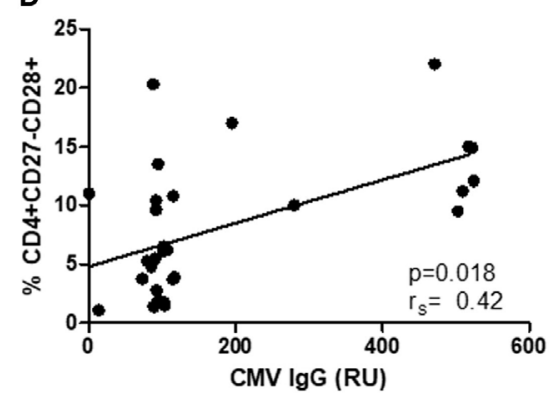

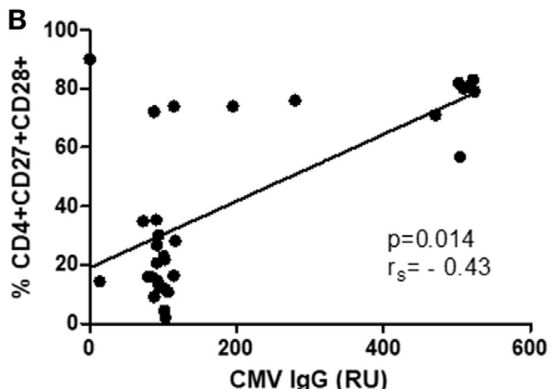

E

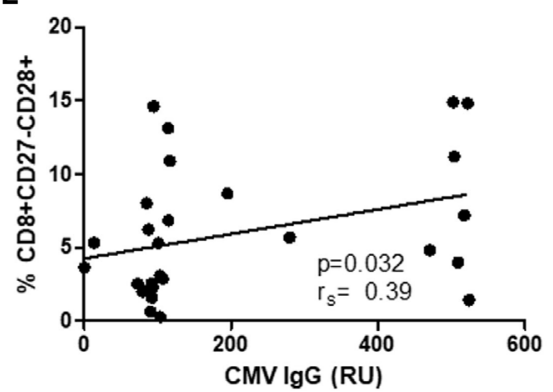

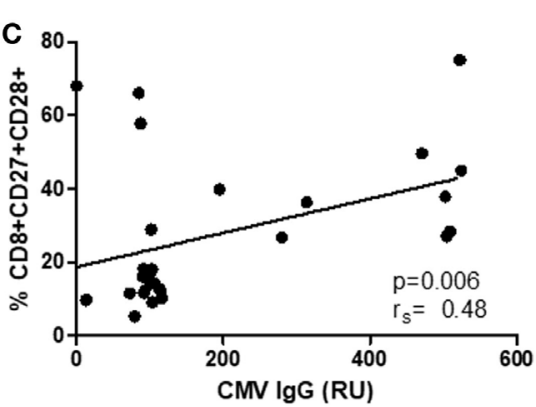

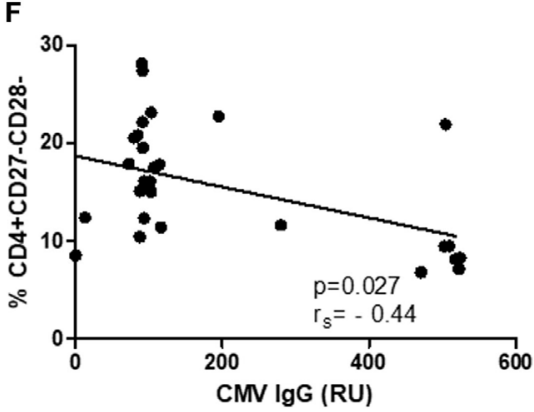

FIGURE 5 | Correlation between IgG anti-cytomegalovirus (CMV) titers and immunosenescence markers in healthy controls. (A-F) Show the correlation between $\mathrm{CD} 3+\mathrm{CD} 56+\left(r_{\mathrm{s}}=0.57, p<0.001\right), \mathrm{CD} 4+\mathrm{CD} 27+\mathrm{CD} 28+\left(r_{\mathrm{s}}=0.43, p=0.01\right), \mathrm{CD} 8+\mathrm{CD} 27+\mathrm{CD} 28+\left(r_{\mathrm{s}}=0.49, p=0.06\right), \mathrm{CD} 4+\mathrm{CD} 27-\mathrm{CD} 28+\left(r_{\mathrm{s}}=0.42\right.$, $p=0.01)$, and CD8+CD27-CD28+ $\left(r_{\mathrm{s}}=0.39, p=0.03\right)$ and anti CMV-IgG titers. 
immune system in a similar way to the effects observed during aging (10). Of note, the expression of KLRG1 was found increased in elderly individuals as well as in sexually abused subjects $(30,31)$. Corroborating this finding, we found an increase in this senescent marker in T-cell subsets of patients, regardless of the history of childhood maltreatment. The PD-1 is another cell-surface marker of late differentiation or exhaustion. The PD-1 and its ligand (PD-L1) have been especially targeted in the area of immunotherapy (checkpoint blockade), given its effectiveness in increasing antitumor response when stimulated by antibodies. The PD-1 is also expressed in most breast tumors $(32,33)$. In support of our findings, a previous study reported the increase of this marker in patients with early stage breast cancer (34). Furthermore, the PD-1 has been also investigated in chronic viral infections (CMV, HIV, and viral hepatitis). Individuals with these infections had a senescent profile, demonstrated by the increased frequency of $\mathrm{PD}-1+$ expressing $\mathrm{T}$ cells $(13,35)$. Considering the intense relationship between viral infections, cancer, and immunosenescence, we can confirm that this profile of cellular senescence and exhaustion is also present in patients with breast cancer.

The CMV has been associated with features of accelerated immunosenescence, including the expansion of senescent $\mathrm{T}$ cells (CD8+CD28-), reduced T-cell repertoire, and increased plasma pro-inflammatory cytokines (IL-6) (12). Furthermore, the presence of acute stressors, such as the diagnosis of cancer, may lead to immune dysregulations implicated with reactivation of latent viruses such as CMV (17). Previously, the presence of fatigue in women with breast cancer undergoing treatment was associated with higher titers of IgG antibodies to CMV (but not EBV) (36). In line, we observed that $\mathrm{CM}+$ group had increased proportion of subjects with higher IgG serology to CMV as compared to $\mathrm{CM}-$ or control groups. During aging studies, the CMV infection has been shown to play a role in driving the expansion of late-differentiated T cells (CD28-) (18), and our data corroborate this hypothesis. In particular, the CMV IgG levels were positively correlated with intermediate- or late-differentiated $\mathrm{T}$ cells as well as negatively correlated with early differentiated $\mathrm{T}$ cells $(\mathrm{CD} 4+\mathrm{CD} 27+\mathrm{CD} 28+)$. We also observed a positive correlation of NK cells with increased CMV IgG titers in women with breast cancer. This result confirms previous data demonstrating that CMV infection can affect innate immunity by expanding the NK cell pool (37). This finding was also observed in individuals without breast cancer (38).

The subgroups of NK cells studied here did not differ between groups, and these data corroborate the finding of Nieto-Velazquez et al. (24). It should be stressed out that the breast cancer patients evaluated here were in early stages, not undergoing treatment. Therefore, the observed results were not influenced by treatment. Witek Janusek et al., on the other hand, have observed that women with breast cancer during treatment (surgery and radiotherapy) with CM history had lower NKCA (22). This divergence can be attributed to the fact that cancer therapy can cause immunomodulatory effects in cellular populations $(24,39)$.
Most changes concerning the expression of activated and regulatory markers were observed in both groups of patients, regardless of the history of childhood maltreatment. Some MFI changes were found in line with reciprocal increase/ decrease in the studied frequency. Indeed, downregulated expression of CD27 and CD28 was observed in PBMCs of patients as compared to controls, in parallel to reduced frequencies of early differentiated T cells (CD27+CD28+) in patients. These two molecules are important co-stimulatory receptors for $\mathrm{T}$ cells, and a reduced expression would be associated with poorer cell-mediated immunity. Although the expression of the CD69 and PD1 did not differ between groups, the $\mathrm{CM}-$ and $\mathrm{CM}+$ patients had similar expansion in the proportion of early activated T cells (CD3+CD69+) and exhausted T cells (CD8+CD27-CD28-PD1+). It should be noted, however, that cell-surface expression (MFI) is not correlated with the frequency of cell subsets expressing these markers. Neither the CD25 expression nor the frequency of early activated $\mathrm{T}$ cells (CD4+CD25+ or CD8+CD25+) differed between groups. However, some alterations were only observed in the CM+ group. Indeed, the expression levels of the regulatory markers NKG2 and CD103 were only found upregulated in $\mathrm{T}$ cells of $\mathrm{CM}+$ patients. To gain further understanding, in vitro studies are necessary to compare unstimulated and stimulated levels of these markers in PBMCs or isolated T cells.

In addition, future studies should confirm the cellular senescence in breast cancer by investigating additional cellular and molecular markers, not studied here, including the p16/p53 pathway (involved with senescence growth arrest), telomere erosion, epigenetic changes, oxidative stress and pro-inflammatory secretome [senescence-associated secretory phenotype (SASP)] (40). A previous study reported that older patients ( $>70$ years) with breast cancer and colorectal cancer had lower percentages of naive and recent thymic emigrants of CD8+ T cells, expanded memory T cells, and shorter PBMC telomeres than age-matched controls (41). Senescent cells can be generated by cancer chemotherapy, potentially fueling aspects of disease progression. In a prospective study with type I to III breast cancer, expression of p16INKa were found increased in CD3 $+\mathrm{T}$ cells immediately following chemotherapy and remained elevated 1 year after treatment (42). However, telomere erosion was not affected by therapy.

Our data should be interpreted considering some limitations. The sample size of women with breast cancer is relatively small and the cross-sectional design may preclude causal relationships. Future prospective studies are necessary to explore whether the senescence-related immune changes are found previous or consequent of the tumor.

To the best of our knowledge, this is the first study assessing immunological markers in early diagnosis breast cancer with history of CM. Our results suggest that the cellular senescence profile is associated to the diagnosis of breast cancer, regardless of the history of childhood abuse. Longitudinal studies are needed to explore the relationship of this senescent profile with clinical progression and treatment response. 


\section{ETHICS STATEMENT}

The study protocol was approved by both scientific and ethics committees (No. 48889815.5.0000.5336 3) of PUCRS (Porto Alegre, Brazil) and written informed consent was obtained from all participants.

\section{AUTHOR CONTRIBUTIONS}

The paper was written by MB, LT, JS, and revised into its final format by all co-authors. Participant recruitment and screening was performed by LT and LB. Flow cytometry panel was setup by LP and LT. Immunophenotyping and cytometric analysis were performed by LP, LT, and MA. Plasma and PBMC isolation were performed by LT and MA. Statistical analysis was performed by LT, JS, and MB. The CMV

\section{REFERENCES}

1. Fagundes CP, Glaser R, Kiecolt-Glaser JK. Stressful early life experiences and immune dysregulation across the lifespan. Brain Behav Immun (2013) 27(1):8-12. doi:10.1016/j.bbi.2012.06.014

2. Coelho R, Viola TW, Walss-Bass C, Brietzke E, Grassi-Oliveira R. Childhood maltreatment and inflammatory markers: a systematic review. Acta Psychiatr Scand (2014) 129(3):180-92. doi:10.1111/acps.12217

3. Hughes K, Bellis MA, Hardcastle KA, Sethi D, Butchart A, Mikton C, et al. The effect of multiple adverse childhood experiences on health: a systematic review and meta-analysis. Lancet Public Health (2017) 2(8):e356-66. doi:10.1016/ S2468-2667(17)30118-4

4. Heim C, Newport DJ, Bonsall R, Miller AH, Nemeroff CB. Altered pituitary-adrenal axis responses to provocative challenge tests in adult survivors of childhood abuse. Am J Psychiatry (2001) 158(4):575-81. doi:10.1176/appi. ajp.158.4.575

5. Carpenter LL, Carvalho JP, Tyrka AR, Wier LM, Mello AF, Mello MF, et al. Decreased adrenocorticotropic hormone and cortisol responses to stress in healthy adults reporting significant childhood maltreatment. Biol Psychiatry (2007) 62(10):1080-7. doi:10.1016/j.biopsych.2007.05.002

6. Bandinelli LP, Levandowski ML, Grassi-Oliveira R. The childhood maltreatment influences on breast cancer patients: a second wave hit model hypothesis for distinct biological and behavioral response. Med Hypotheses (2017) 108:86-93. doi:10.1016/j.mehy.2017.08.007

7. Goldsmith RE, Jandorf L, Valdimarsdottir H, Amend KL, Stoudt BG, Rini C, et al. Traumatic stress symptoms and breast cancer: the role of childhood abuse. Child Abuse Negl (2010) 34(6):465-70. doi:10.1016/j.chiabu.2009.10.007

8. Elwenspoek MMC, Kuehn A, Muller CP, Turner JD. The effects of early life adversity on the immune system. Psychoneuroendocrinology. 82:140-154. 2017. doi:10.1016/J.PSYNEUEN.2017.05.012

9. Heikkinen S, Miettinen J, Pukkala E, Koskenvuo M, Malila N, Pitkaniemi J. Impact of major life events on breast-cancer-specific mortality: a case fatality study on 8000 breast cancer patients. Cancer Epidemiol (2017) 48:62-9. doi:10.1016/j.canep.2017.03.008

10. Morey JN, Boggero IA, Scott AB, Segerstrom SC. Current directions in stress and human immune function. Curr Opin Psychol (2015) 5:13-7. doi:10.1016/j. copsyc.2015.03.007

11. Crosswell AD, Bower JE, Ganz PA. Childhood adversity and inflammation in breast cancer survivors. Psychosom Med (2014) 76(3):208-14. doi:10.1097/ PSY.0000000000000041

12. Bauer ME, Fuente Mde L. The role of oxidative and inflammatory stress and persistent viral infections in immunosenescence. Mech Ageing Dev (2016) 158:27-37. doi:10.1016/j.mad.2016.01.001

13. Xu W, Larbi A. Markers of T cell senescence in humans. Int J Mol Sci (2017) 18(8):E1742. doi:10.3390/ijms18081742

14. Tavenier J, Langkilde A, Haupt TH, Henriksen JH, Jensen FK, Petersen J, et al. Immunosenescence of the CD8(+) T cell compartment is associated serology was determined by BLC. The study was conceived by RG-O and MB. All authors read and approved the final manuscript.

\section{FUNDING}

We are very grateful to the patients and staff at the Hospital São Lucas (Porto Alegre, Brazil). This work was supported by grants from Conselho Nacional de Desenvolvimento Científico e Tecnológico (CNPq), National Institute of Science and Technology on Neuroimmunomodulation (INCT-NIM), Coordenação de Aperfeiçoamento de Pessoal de Nível Superior (CAPES), and Fundação de Amparo à Pesquisa do Estado do Rio Grande do Sul (FAPERGS). These funding bodies played no role in the writing of this manuscript, nor in the design, data collection, analysis, and interpretation of our data.

with HIV-infection, but only weakly reflects age-related processes of adipose tissue, metabolism, and muscle in antiretroviral therapy-treated HIV-infected patients and controls. BMC Immunol (2015) 16:72. doi:10.1186/s12865015-0136-6

15. Appay V, Dunbar PR, Callan M, Klenerman P, Gillespie GM, Papagno L, et al. Memory CD8+ T cells vary in differentiation phenotype in different persistent virus infections. Nat Med (2002) 8(4):379-85. doi:10.1038/nm0402-379

16. Appay V, Bosio A, Lokan S, Wiencek Y, Biervert C, Kusters D, et al. Sensitive gene expression profiling of human $\mathrm{T}$ cell subsets reveals parallel post-thymic differentiation for CD4+ and CD8+ lineages. J Immunol (2007) 179(11):7406-14. doi:10.4049/jimmunol.179.11.7406

17. Janicki-Deverts D, Cohen S, Doyle WJ, Marsland AL, Bosch J. Childhood environments and cytomegalovirus serostatus and reactivation in adults. Brain Behav Immun (2014) 40:174-81. doi:10.1016/j.bbi.2014.03.010

18. Sylwester AW, Mitchell BL, Edgar JB, Taormina C, Pelte C, Ruchti F, et al. Broadly targeted human cytomegalovirus-specific CD4+ and CD8+ T cells dominate the memory compartments of exposed subjects. J Exp Med (2005) 202(5):673-85. doi:10.1084/jem.20050882

19. Grassi-Oliveira R, Cogo-Moreira H, Salum GA, Brietzke E, Viola TW, Manfro GG, et al. Childhood trauma questionnaire (CTQ) in Brazilian samples of different age groups: findings from confirmatory factor analysis. PLoS One (2014) 9(1):e87118. doi:10.1371/journal.pone.0087118

20. Levandowski ML, Viola TW, Prado CH, Wieck A, Bauer ME, Brietzke E, et al. Distinct behavioral and immunoendocrine parameters during crack cocaine abstinence in women reporting childhood abuse and neglect. Drug Alcohol Depend (2016) 167:140-8. doi:10.1016/j.drugalcdep.2016.08.010

21. Beck AT, Steer RA, Ball R, Ranieri W. Comparison of beck depression inventories -IA and -II in psychiatric outpatients. JPers Assess (1996) 67(3):588-97. doi:10.1207/s15327752jpa6703_13

22. Witek Janusek L, Tell D, Albuquerque K, Mathews HL. Childhood adversity increases vulnerability for behavioral symptoms and immune dysregulation in women with breast cancer. Brain Behav Immun (2013) 30(Suppl):S149-62. doi:10.1016/j.bbi.2012.05.014

23. Brown MJ, Thacker LR, Cohen SA. Association between adverse childhood experiences and diagnosis of cancer. PLoS One (2013) 8(6):e65524. doi:10.1371/journal.pone.0065524

24. Nieto-Velazquez NG, Torres-Ramos YD, Munoz-Sanchez JL, EspinosaGodoy L, Gomez-Cortes S, Moreno J, et al. Altered expression of natural cytotoxicity receptors and NKG2D on peripheral blood NK cell subsets in breast cancer patients. Transl Oncol (2016) 9(5):384-91. doi:10.1016/j. tranon.2016.07.003

25. Tsuda B, Miyamoto A, Yokoyama K, Ogiya R, Oshitanai R, Terao M, et al. B-cell populations are expanded in breast cancer patients compared with healthy controls. Breast Cancer (2018) 25(3):284-91. doi:10.1007/s12282-017-0824-6

26. Song G, Wang X, Jia J, Yuan Y, Wan F, Zhou X, et al. Elevated level of peripheral CD8(+)CD28(-) T lymphocytes are an independent predictor of progression-free survival in patients with metastatic breast cancer during the 
course of chemotherapy. Cancer Immunol Immunother (2013) 62(6):1123-30. doi:10.1007/s00262-013-1424-8

27. Meloni F, Morosini M, Solari N, Passadore I, Nascimbene C, Novo M, et al. Foxp3 expressing CD4+ CD25+ and CD8+CD28- T regulatory cells in the peripheral blood of patients with lung cancer and pleural mesothelioma. Hum Immunol (2006) 67(1-2):1-12. doi:10.1016/j.humimm.2005.11.005

28. Chen IH, Lai YL, Wu CL, Chang YF, Chu CC, Tsai IF, et al. Immune impairment in patients with terminal cancers: influence of cancer treatments and cytomegalovirus infection. Cancer Immunol Immunother (2010) 59(2):323-34. doi:10.1007/s00262-009-0753-0

29. Onyema OO, Decoster L, Njemini R, Forti LN, Bautmans I, De Waele M, et al. Chemotherapy-induced changes and immunosenescence of CD8+ T-cells in patients with breast cancer. Anticancer Res (2015) 35(3):1481-9.

30. Henson SM, Akbar AN. KLRG1 - more than a marker for T cell senescence. Age (Dordr) (2009) 31(4):285-91. doi:10.1007/s11357-009-9100-9

31. Kared H, Martelli S, Ng TP, Pender SL, Larbi A. CD57 in human natural killer cells and T-lymphocytes. Cancer Immunol Immunother (2016) 65(4):441-52. doi:10.1007/s00262-016-1803-z

32. Bedognetti D, Maccalli C, Bader SB, Marincola FM, Seliger B. Checkpoint inhibitors and their application in breast cancer. Breast Care (Basel) (2016) 11(2):108-15. doi:10.1159/000445335

33. Hu X, Huang W, Fan M. Emerging therapies for breast cancer. J Hematol Oncol (2017) 10(1):98. doi:10.1186/s13045-017-0466-3

34. Poschke I, De Boniface J, Mao Y, Kiessling R. Tumor-induced changes in the phenotype of blood-derived and tumor-associated T cells of early stage breast cancer patients. Int J Cancer (2012) 131(7):1611-20. doi:10.1002/ijc.27410

35. Gianesin K, Noguera-Julian A, Zanchetta M, Del Bianco P, Petrara MR, Freguja R, et al. Premature aging and immune senescence in HIV-infected children. AIDS (2016) 30(9):1363-73. doi:10.1097/QAD.0000000000001093

36. Fagundes CP, Glaser R, Alfano CM, Bennett JM, Povoski SP, Lipari AM, et al. Fatigue and herpesvirus latency in women newly diagnosed with breast cancer. Brain Behav Immun (2012) 26(3):394-400. doi:10.1016/j. bbi.2011.09.014
37. Mozaffari F, Lindemalm C, Choudhury A, Granstam-Bjorneklett H, Helander I, Lekander M, et al. NK-cell and T-cell functions in patients with breast cancer: effects of surgery and adjuvant chemo- and radiotherapy. Br J Cancer (2007) 97(1):105-11. doi:10.1038/sj.bjc.6603840

38. Ostapchuk YO, Cetin EA, Perfilyeva YV, Yilmaz A, Skiba YA, Chirkin AP, et al. Peripheral blood NK cells expressing HLA-G, IL-10 and TGF-beta in healthy donors and breast cancer patients. Cell Immunol (2015) 298(1-2):37-46. doi:10.1016/j.cellimm.2015.09.002

39. Mamessier E, Pradel LC, Thibult ML, Drevet C, Zouine A, Jacquemier J, et al. Peripheral blood NK cells from breast cancer patients are tumor-induced composite subsets. J Immunol (2013) 190(5):2424-36. doi:10.4049/jimmunol. 1200140

40. McHugh D, Gil J. Senescence and aging: causes, consequences, and therapeutic avenues. J Cell Biol (2018) 217(1):65-77. doi:10.1083/jcb.201708092

41. Falci C, Gianesin K, Sergi G, Giunco S, De Ronch I, Valpione S, et al. Immune senescence and cancer in elderly patients: results from an exploratory study. Exp Gerontol (2013) 48(12):1436-42. doi:10.1016/j.exger.2013.09.011

42. Sanoff HK, Deal AM, Krishnamurthy J, Torrice C, Dillon P, Sorrentino J, et al. Effect of cytotoxic chemotherapy on markers of molecular age in patients with breast cancer. J Natl Cancer Inst (2014) 106(4):dju057. doi:10.1093/jnci/ dju057

Conflict of Interest Statement: The authors declare that the research was conducted in the absence of any commercial or financial relationships that could be construed as a potential conflict of interest.

Copyright () 2018 Trintinaglia, Bandinelli, Grassi-Oliveira, Petersen, Anzolin, Correa, Schuch and Bauer. This is an open-access article distributed under the terms of the Creative Commons Attribution License (CC BY). The use, distribution or reproduction in other forums is permitted, provided the original author(s) and the copyright owner(s) are credited and that the original publication in this journal is cited, in accordance with accepted academic practice. No use, distribution or reproduction is permitted which does not comply with these terms. 